Bulletin of the AAS • Vol. 53, Issue 2

\title{
Astronomers for Planet Earth: Engaging with the Public to Forge a Sustainable Future
}

J. A. White ${ }^{1}$, H. Dalgleish ${ }^{2}$, L. Burtscher ${ }^{3}$, A. J. Frost ${ }^{4}$, T. Beuchert $^{5}$, The A4E Community 6

${ }^{1}$ National Radio Astronomy Observatory, ${ }^{2}$ University of Namibia, ${ }^{3}$ Sterrewacht Leiden, ${ }^{4}$ Institute of Astronomy, KU Leuven, ${ }^{5}$ European Southern Observatory, ${ }^{6}$ Earth

Published on: Apr 09, 2021

License: Creative Commons Attribution 4.0 International License (CC-BY 4.0). 


\begin{abstract}
The climate crisis is here and the time for action is ticking away. Scientific evidence has repeatedly shown that humanity alone is responsible for the destructive changes happening to our climate. Likewise, the responsibility to address and curb global warming lies with us all. As astronomers, we have a unique opportunity to educate people on the impacts of anthropogenic climate change and urge those in power to take meaningful action. Astronomers for Planet Earth is a group of current and former professional astronomers, amateur astronomers, astronomy educators, and astronomy students from over 40 countries around the world. The organisation's goal is to 1) provide astronomers interested in climate change with a community to discuss ideas; 2) provide the public with information about the climate crisis; and 3) provide the climate movement with an astronomical perspective.
\end{abstract}

\title{
The Climate Crisis
}

The window to slow down anthropogenic climate change is quickly closing [1]]. The required reduction in greenhouse gas emissions necessary to limit global warming as outlined in the Paris Agreement [2] — is not being met. Our global society is currently on track to exceed the total carbon emissions designed to keep the Earth below $1.5^{\circ} \mathrm{C}$ global warming [ $\underline{3}$ ]. The responsibility to take action and curb emissions is in our hands.

As well as posing a threat to humanity and our way of life, the climate crisis will impact our ability to conduct astronomical research. Changes in weather patterns due to climate change are already taking a toll on ground-based astronomical observations [4]. Rising temperatures and a corresponding increase in atmospheric water vapor content are leading to lower quality observing conditions and show no sign of abating. The carbon footprint of the average astronomer is much larger than both that of the average person and academics in other fields [ㄷ]. Our high emissions are in large part due to the nature of the field including: operational impacts of observatories in remote areas []]; high performance computational needs []]; and frequent travel (often by flying) to conferences []ㅡ.

The carbon emissions of our field must be reconciled with the guidelines outlined in the Paris Agreement [2]]. Our ability to conduct astronomy research in a carbon constrained future relies on changes that we make now. As stated in []], to futureproof astronomy, we must recognize impending environmental change, financial 
uncertainties, and the need for moral introspection, all of which threaten to hinder the continuation of the discipline.

Astronomers are uniquely positioned as researchers, educators, and active facilitators of public outreach []․ We share a great privilege in reaching and inspiring children, students, and the general public. In order to keep our field thriving and flourishing in the future, we must increase our efforts to communicate the seriousness and impacts of the impending climate crisis today.

\section{Astronomers for Planet Earth}

Astronomers have a unique perspective of Earth. We are acutely aware of the vast distances between us and other planetary systems. While travel to habitable exoplanets may someday be possible, the required timescales are such that it is not feasible within our lifetimes or anywhere in the near future.

Astronomers for Planet Earth (A4E) was founded to bring our unique position to the fore. A4E is a volunteer network of current and former professional astronomers, astronomy students, astronomy educators, amateur astronomers, and astronomy enthusiasts committed to bringing our voices to the struggle against climate change and for climate justice. A4E was originally founded in 2019 with the merger of a US and European group of astronomers that shared a similar vision. The group has since grown to include members from many countries world-wide.

A4E has three primary goals. These are to provide:

1. astronomers interested in addressing the climate crisis with a community, in order to facilitate discussion and sharing of resources

2. the public with information about the climate crisis

3. the climate movement with an astronomical perspective

We currently have more than 800 members [10], hailing from 40 countries: Argentina, Australia, Austria, Azerbaijan, Belgium, Brazil, Bulgaria, Canada, Chile, China, Colombia, Czech Republic, Denmark, Dominican Republic, Finland, France, Germany, Hungary, India, Iran, Ireland, Italy, Japan, Kenya, Mexico, Netherlands, New Zealand, Nigeria, Pakistan, Philippines, Portugal, Serbia, South Africa, Spain, Sweden, Switzerland, Turkey, United Kingdom, United States, and Uruguay. 


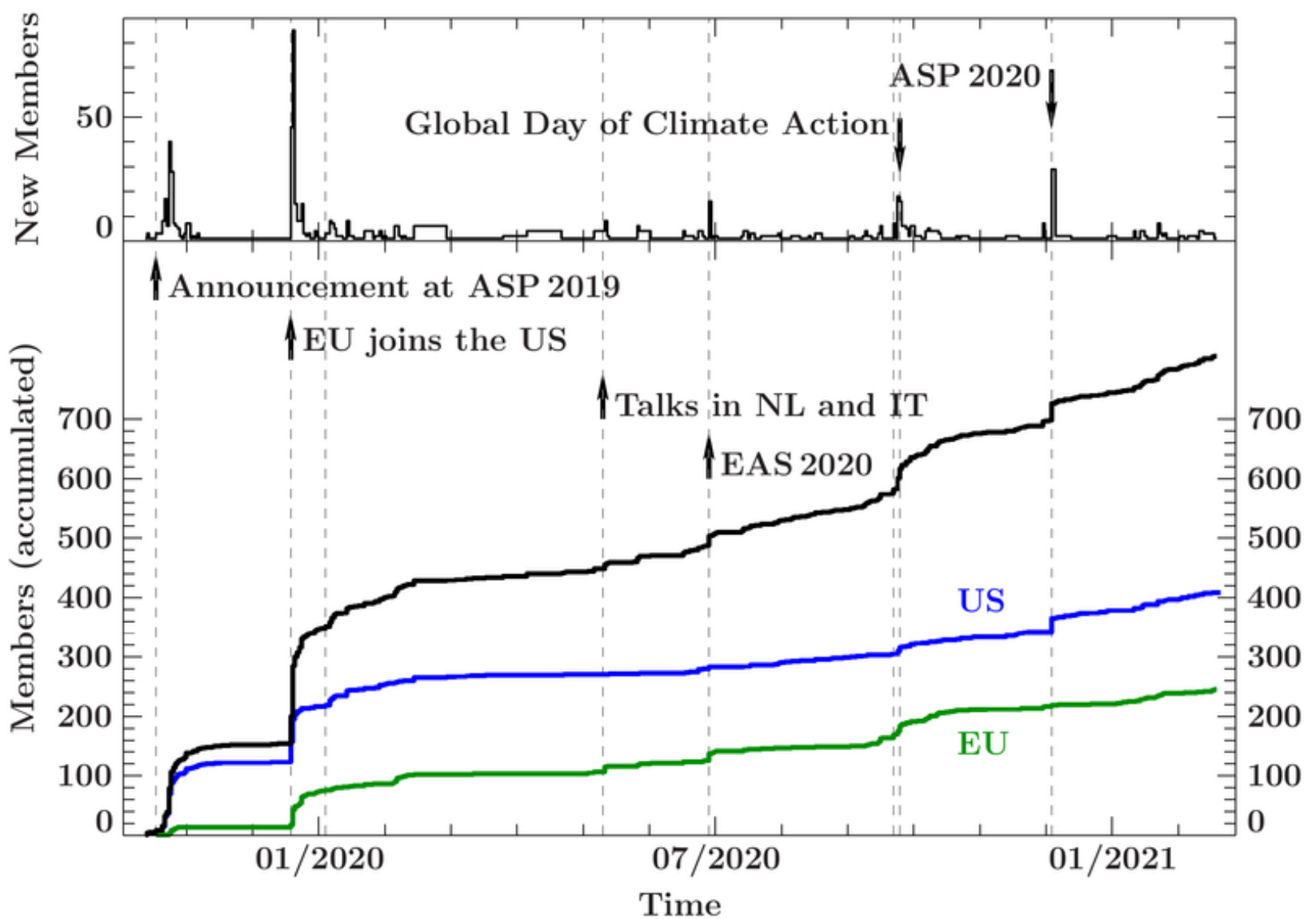

Figure 1. Cumulative A4E member count as a function of time. The black curve is the total membership, the blue curve denotes the members from the US, and the green curve denotes members from within the EU. Events that led to a significant spike in membership, such as talks by A4E members or conferences in which A4E was involved, are denoted as well.

\section{A4E Benefits and activities}

A4E membership is free and provides many benefits. These include access to our webinars, Slack workspace, Google Drive, mailing list, newsletter, and a great range of resources, as well as open invitations to working groups and their associated online meetings.

The regular webinars include discussions with invited experts on the intersection of astronomy and the climate crisis. Past topics have included: the link between fossil fuels and rising temperatures; changing careers to focus on sustainability; and how climates have evolved on the other planets in the solar system. The target audience for the webinars is A4E members (as opposed to the general public) and they are typically 20-40 minutes in length. A recent webinar with Dr. Faustine Cantalloube (MPIA, Heidelberg, Germany) discusses the impact of climate change on astronomy: increased 
temperatures and changed weather patterns are already affecting operations of the European Southern Observatory in Chile. A4E members have exclusive access to the videos before they are indexed on YouTube in addition to the live Q\&A session with the speaker through Zoom.

Making use of the broad range of talent within the A4E community, members have produced original videos that highlight our cause and discuss the severity of the climate crisis. These videos are hosted on $\underline{\text { Vimeo, }} \underline{\text { YouTube, }}$ and are also indexed on the A4E website. Teaching materials, public outreach talks, and other climate change related resources are kept on our Google Drive. Members can request access to the Drive and are free to use any of the resources in their teaching or outreach.

We also welcome members to participate in our working groups, which focus on key aspects of our organization. The scope ranges from PR - where social media strategies are discussed and implemented, to Operations - where the logistics and planning of A4E primarily takes place. The working groups are organized through Slack alongside regular meetings. Slack is a major communication hub for A4E members; there are channels for everything from regional news, to teaching resources, to discussing light pollution. Our Slack workspace is exclusive to A4E members and a link to join is sent after membership is confirmed. If you are interested in becoming a member and/or joining or proposing a working group, please see Section $\underline{4}$.

\section{Membership and how to join}

Are you a current or former:

- student of astronomy or planetary science?

- astronomy educator?

- amateur astronomer or citizen scientist?

- postdoc, researcher, or professor of astronomy or planetary science?

- astronaut or space scientist?

- science writer or science fiction writer who writes about astronomy?

- observatory operator or someone whose work is closely tied to astronomy?

If you are, do you also agree that:

- climate change is caused by human activity?

- climate change is urgent, and needs immediate action?

- there is value in bringing an astronomical perspective to education and outreach on this issue? 
Then join us! A4E members receive access to our groups.io page, webinar Q\&A, mailing list, Slack workspace, and Google Drive resources. Please visit our webpag $\underline{\text { e }}$ to join. There is no commitment required and you can be as engaged as you choose to be.

Acknowledgements: We are grateful for the immense efforts of all the A4E members. We have achieved a significant amount in a very short space of time and we look forward to the exciting progress to come. Thank you all for joining us on this important mission.

\section{Citations}

1. Emissions Gap Report 2020 (United Nations Environment Programme, 2020); https://www.unep.org/emissions-gap-report-2020

2. The Paris Climate Accord (UNFCCC, 2016); https://unfccc.int/process-and$\underline{\text { meetings/the-paris-agreement/the-paris-agreement }} \triangleq$

3. IPCC Special Report on Global Warming of 1.5 C (WMO, 2018); https://www.ipcc.ch/sr15/

4. Cantalloube, F., Milli, J., Böhm, C. et al., 2020, Nat. Astron., 4, 826-829

5. Dalgleish, H., 2020, Astron. \& Geo., 61, 6, 18-21

6. Flagey, N., Thronas, K., Petric, A., et al., 2020, Nat. Astron., 4, 816-818.

7. Stevens, A.R.H., Bellstedt, S., Elahi, P.J. et al., 2020, Nat. Astron., 4, 843-85

8. Burtscher, L., Barret, D., Borkar, A.P. et al., 2020, Nat. Astron., 4, 823-825

9. Entradas, M., Bauer, M. W., 2019, Nat. Astron., 3, 183-187.

10. Astronomers for Planet Earth (2021); https://astronomersforplanet.earth/about-

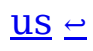

